\title{
Replacing the Fourier transformation in optical coherence tomography with multiple comparison operations
}

\author{
Adrian Bradu ${ }^{\mathrm{a}}$, Sylvain Rivet ${ }^{\mathrm{b}}$, Adrian Podoleanu $^{\mathrm{a}}$ \\ a Applied Optics Group, School of Physical Sciences, University of Kent, Canterbury CT2 7NH, UK \\ bUniversité de Bretagne Occidentale, EA 938 Laboratoire de Spectrométrie et Optique Laser, 6 \\ avenue Le Gorgeu, C.S. 93837, 29238 Brest Cedex 3, France \\ *a.bradu@kent.ac.uk
}

\section{ABSTRACT}

The conventional spectral domain (SD) and Fourier domain (FD) OCT method deliver a 1D reflectivity profile in the sample investigated by applying a Fourier transform (FT) to the channeled spectrum, CS, at the interferometer output. We discuss here the advantages of a novel OCT technology, Master Slave (MS). The MS method radically changes the main building blocks of a SD (FD)-OCT set-up. The serially provided electrical signal in conventional technology is replaced by multiple signals, a signal for each OPD point along an electrical output for each depth in the object investigated. In this way, it is possible to: (1) direct access to information from selected depths; (ii) eliminate the process of resampling, required by the FT based conventional technology, with immediate consequences in improving the decay of sensitivity with depth, achieving the expected axial resolution limit, reduction in the time to display an image and lower cost OCT assembly; (iii) OCT interferometer tolerant to dispersion left unbalanced.

\section{INTRODUCTION}

Both implementations of the Fourier domain optical coherence tomography, spectrometer based (Sp)-OCT and swept source (SS)-OCT, can be used to produce both cross-sectional (B-scan) and en-face (C-scan) images with high resolution and high sensitivity.

Traditionally, in order to produce a volumetric image, in both implementations, each channeled spectrum acquired while scanning the probing beam over the sample is subject to a fast Fourier transform (FFT). However, before the FFT, several preparatory signal processing steps such as zero padding, spectral shaping, apodization, dispersion compensation or data resampling are required to produce high axial resolution and sensitivity images. As all these steps are sequentially executed and require time, therefore, the need to perform them impact production of the images in real-time.

So far, several techniques involving both hardware and/or software solutions have been demonstrated to successfully eliminate or diminish the execution time of the preparatory steps. Correct resampling and compensation for dispersion mismatch are extremely important as an incorrect k-mapping or dispersion left unbalanced in the system generates a broadening of the coherence peak. To eliminate the resampling step, in Sp-OCT a solution using a prism after the diffraction grating was proposed. However, this complicates the optics hardware, requires careful hardware adjustment of several components, introduces losses and the resampling is prone to errors. In SS-OCT, the swept sources are often equipped with a supplementary clock signal (k-clock) that adds not only to the cost of the source, but requires a specialized, more sophisticated digitizer that in the end does not warranty a perfect resampling and also limits the axial range of the image. Other techniques such as using an additional light source, parametric iteration methods, phase linearization techniques, and automatic calibrations have been also proposed.

All these methods are normally computationally expensive and limit the real-time operation of the OCT systems. Dispersion compensation is another important limiting factor in obtaining high-resolution SD (or FD)-OCT images. We will refer from now on to both SD and FD methods as to SD only. Both hardware and software methods were implemented to overcome this limitation. Various numerical techniques to compensate for dispersion have been developed. As all the numerical algorithms used to compensate for dispersion involve Hilbert transformations, phase corrections, filtering, etc., a quite heavy computational loading and therefore numerical dispersion compensation has to be performed as a postprocessing process. 
The development of solutions involving the utilization of compute unified device architecture (CUDA) that enables dramatic increases in computing performance by using graphic processing units (GPU)s or field programmable gate array (FPGA) solutions lead to a tremendous reduction in the computation time of the preparatory steps. However, as some of the preliminary steps are often based on iterative calculations, errors in correctly preparing the data are unavoidable.

The method we are proposing, Master/Slave (MS), does not require any FFT, hence the preparatory steps (resampling and compensation for dispersion mismatch between the two arms of the interferometer) are not required. Thus, the MS method being based on a pure mathematical operation only, is able in principle to produce better images in terms of their axial resolution and sensitivity than their FFT based counterpart. Moreover, as MS-OCT involves mathematical operations that can be performed in parallel, it makes sense to take advantage of GPU or FPGA parallelizable methods to potentially achieve true real-time generation of OCT images.

We discuss here the advantages of the novel MS-OCT technology [1-8]. The MS technique delivers a single reflectivity point from a selected depth, by comparing the acquired channeled spectrum (CS) with many replicas of the CS (masks). To compete with the conventional FT based technology, Nz replicas (masks) of the CS are generated. The MS procedure is performed by comparing the CS obtained with the sample in place, with $\mathrm{N}_{z}$ masks, employing a comparator for each mask. In this way, $\mathrm{N}_{\mathrm{z}}$ signals along $\mathrm{N}_{\mathrm{z}}$ separate wires are produced, for the $\mathrm{N}_{\mathrm{z}}$ points of the A-scan, where all signals are delivered in parallel. Thus, the MS method radically changes the main building blocks of a SD (FD)-OCT set-up. The serially provided signal in conventional technology is replaced by multiple signals, a signal for each OPD point along a line in depth in the object investigated.

\section{PROCEDURES OF PRODUCING IMAGES IN OCT}

In this manuscript, we will refer to an improvement to the MS method, recently reported, complex master/slave (CMS) [1].

\subsection{Strategies of producing reflectivity profiles in OCT}

To obtain the reflectivity profile, $A(z)$, at a certain axial position $(z)$ in the A-scan, the integral of the product between the channeled spectrum $C S\left(\varphi_{k, z}\right)$ from the sample and the kernel function $e^{j k z}$ has to be calculated, where $\varphi_{k, z}$ is the phase of the measured channeled spectrum, while $k$ is its wavenumber.

$$
\mathrm{A}(\mathrm{z})^{\text {chirped }}=\int_{-\infty}^{+\infty} \mathrm{CS}\left(\varphi_{\mathrm{k}, \mathrm{z}}\right) \cdot \mathrm{e}^{\mathrm{jkz}} \mathrm{dk}
$$

Due to nonlinearities in the decoding procedure, from spectrum to time, typical for a real spectrometer or tuning swept source, the channeled spectrum is chirped. This means that the spectrum modulation does not exhibit a regular periodicity over the wavenumber axis. A second source of nonlinearity in wavenumber is that due to dispersion in the interferometer. Therefore, the phase is not directly proportional to $k$, but expressed in a general way as:

$$
\varphi_{k, z}=g_{k} z+h_{k}
$$

The parameters $g_{k}$ and $h_{k}$ take into account the non-linear dependence of the phase on the wavenumber and on dispersion left unbalanced in the interferometer respectively [1].

Let us compare two strategies of producing reflectivity profiles in OCT: FT based (conventional) and CMS based.

\subsubsection{Conventional FT based OCT}

The chirp determined by $g_{k}$ and $h_{k}$ deviate the CS from being represented as a pure sinusoidal component, pulsating at a given periodicity. Therefore, if conventional method is used, the FT leads to a wide peak with shoulders. To eliminate the chirp, the conventional FT based technology requires resampling of the CS. The kernel function $e^{j k z}$ is not modified. Each $\mathrm{CS}$ is first resampled then multiplied with a function that cancels the effect of the dispersion to obtain a linear relationship between the phase of the modified channeled spectrum $\left(\mathrm{CS}^{\text {non-chirped }}\right)$ and $k$. As a result, an accurate A-scan is produced by performing a FFT of the $\mathrm{CS}^{\text {non-chirped: }}$

$$
\mathrm{A}(\mathrm{z})^{\mathrm{non}-\text { chirped }}=\int_{-\infty}^{+\infty} \mathrm{CS}^{\text {non-chirped }}(\mathrm{k}) \cdot \mathrm{e}^{\mathrm{jkz}} \mathrm{dk}=\operatorname{FFT}\left[\operatorname{CS}^{\text {non-chirped }}(\mathrm{k})\right]
$$


The advantage of this approach is the speed of the FFT. However, the preparatory steps to correct the data and produce $\mathrm{CS}^{\text {non-chirped }}$ before performing FFT are computationally time expensive and prone to introducing errors. Moreover, the phase correction procedure has to be done for each acquired channeled spectrum (i.e. for each lateral pixel), as illustrated in Fig. 1.

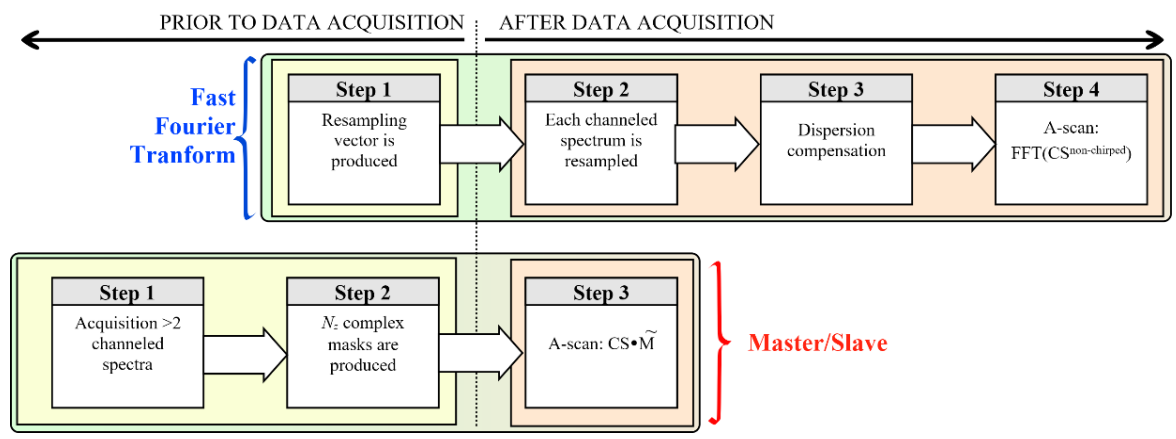

Fig. 1. Procedure steps required to produce an A-scan profile using the FT based OCT (top) and procedures steps required to produce a single reflectivity value using the CMS method (bottom).

\subsubsection{MS based OCT}

The MS strategy consists in modifying the kernel function $e^{j k z}$ in Eq. (1) rather than process the CS. To produce a single point in the A-scan, an integral of the product between the chirped CS and a new kernel function $\tilde{M}\left(\varphi_{k, z}\right)$ (denoted as complex masks) is performed. As reflectivity of a single point only is delivered by such an integral calculation, the MS method may not present the speed advantage of the FFT based conventional OCT technology (that delivers information from all depth in a single FFT step).; however, the MS method eliminates some disadvantages of the FFT method. The improved version of the MS-OCT recently reported, the CMS, allows calculation of complex masks $\tilde{M}\left(\varphi_{k . z}\right)$, represented as arrays of components $\left\{M\left(\varphi_{1, z}\right), M\left(\varphi_{2, z}\right), \ldots M\left(\varphi_{N_{k}, z}\right)\right\}$, with a component for each wavenumber pixel $1,2, \ldots N_{k}$ along the coordinate $k$. In order to obtain the reflectivity from an axial position $z$, it is sufficient to calculate:

$$
\mathrm{A}(\mathrm{z})^{\mathrm{non}-\text { chirped }}=\int_{-\infty}^{+\infty} \operatorname{CS}\left(\varphi_{\mathrm{k}, \mathrm{z}}\right) \cdot \frac{\partial \mathrm{g}}{\partial \mathrm{k}} \mathrm{e}^{\mathrm{j} \varphi_{\mathrm{k}, \mathrm{z}} \mathrm{dk}}=\int_{-\infty}^{+\infty} \operatorname{CS}\left(\varphi_{\mathrm{k}, \mathrm{z}}\right) \cdot \tilde{\mathrm{M}}\left(\varphi_{\mathrm{k}, \mathrm{z}}\right) \mathrm{dk}
$$

As illustrated in Fig. 1, the only step to be performed after data acquisition is the dot product between the recorded CS and the complex masks.

\subsection{Procedures of producing cross-section images in OCT}

In the diagram shown in Fig. 1, the complete procedures of producing A-scans, using the FT-OCT (top) and CMS (bottom) are presented. Both techniques require a "calibration" step, before data acquisition, where the FT method entails three operations, which can only be executed sequentially whilst the CMS entails a single operation as described below.

\subsubsection{Conventional FT based OCT}

Let $k=1 \ldots N_{k}$ be the number of wavenumber sampled points used to digitize each channeled spectrum, for the axial positions $z=1 \ldots N_{z}$ where the reflectivities are to be evaluated from and let us consider $x=1 \ldots N_{x}$, the number of Ascans in each B-scan. An A-scan is produced as $F F T\left(C S_{x}^{\text {non-chirped }}\right)$. Therefore, mathematically, a B-scan image can be described by:

$$
B_{F F T}=\left[\begin{array}{c}
F F T\left(C S_{1}^{\text {non-chirped }}\right) \\
F F T\left(C S_{2}^{\text {non-chirped }}\right) \\
\vdots \\
F F T\left(C S_{N_{x}}^{\text {non-chirped }}\right)
\end{array}\right]
$$


where each of the components, $C S_{x}^{\text {non-chirped }}$, for $x=1 . . N_{x}$, are resampled channeled spectra of $N_{k}$ components each, recorded while scanning the beam over the sample. The size in pixels of the image thus obtained is $\left(N_{x} \times N_{k}\right) / 2\left(N_{x}\right.$ pixels laterally and $N_{k} / 2$ pixels axially). To produce a cross-sectional image using conventional FTs:

1. A recalibration vector is produced. This is a step performed prior to data acquisition.

2. The recalibration vector is used to resample each channeled spectrum via a spline cubic interpolation (not required for swept sources equipped with k-clocks). Obviously, this step takes place during or after data acquisition.

3. A procedure to compensate dispersion left unbalanced in the interferometer. This step can be performed during or after data acquisition.

4. A B-scan is produced by performing FFTs of the channeled spectra modified according to the steps 2 and 3 above.

The time required to produce a B-scan in FT based OCT includes the timing of sequentially executing the 3 last steps presented above. For swept sources equipped with k-clocks signals, the steps 1 and 2 can be eliminated.

\subsubsection{MS based OCT}

Eq. (4) can be rewritten to describe the reflectivity value from a scattering center at an axial position $z$, by:

$$
A_{z}=\sum_{k=1}^{N_{k}} C S\left(\varphi_{k, z}\right) \tilde{M}(k, z)
$$

A B-scan image can be represented in a matrix form as:

$$
B_{C M S I}=C S \times \tilde{M}
$$

where CS is a matrix of size $N_{x} \times N_{k}$, containing the channeled spectra $C S_{x}$, acquired for all lateral pixels $x=1,2, \ldots N_{x}$ (along a laterally oriented scan). Each row of matrix $\tilde{M}$ is a complex signal of $N_{k}$ components representing a mask produced for each axial position $z=1,2, \ldots N_{z}$. The CS used in Eq. (4) to produce the B-scans, do not require any preparation, while the resulting B-scan images are completely free of eventual unbalanced dispersion or decoding nonlinearities.

A CMS based cross-sectional image is generated according to the following procedure:

1. With a high reflector as object, two or more experimental channeled spectra corresponding to different axial positions are stored. This step is performed only once for a given experimental set-up, before data acquisition.

2. The experimental channeled spectra recorded at the step above are then used to theoretically infer $N_{z}$ complex masks [1]. This step, that is performed before data acquisition, needs to be repeated only if the axial range displayed in the B-scan image is modified.

3. A cross-sectional image is produced by multiplying two matrices (Eq. 7). This step is performed as soon as raw data corresponding to a B-scan is acquired.

The size in pixels of the cross-sectional MS-OCT image produced in Eq. (7) is $N_{x} \times N_{z}$, different from that described by Eq. (5) for the FT case, which is $N_{x} \times N_{k} / 2$. This axial range difference triggers a discussion on an important aspect: when using the conventional strategy, FFT based, the axial range of each A-scan scales from $z_{\min }=0$ to a maximum value $z_{\max }$, determined by the sampling speed of the digitizer, hence by the number of sampling points $N_{k}$ used to digitize the channeled spectrum. A modification of the axial range of interest (ROI), if needed, can only be achieved by effectively cropping the cross-section image, while a modification of the number of sampling points of the ROI is only possible by zero-padding the channeled spectra before FFT. In CMS, the axial length of the axial region of interest ROI is completely independent on the number of digitized points $\mathrm{N}_{\mathrm{k}}$. In addition, the axial range of interest as well as its coverage is adjustable by selecting the set of complex masks $\tilde{M}$ in terms of their axial position and increment between their depths.

The time required to produce a CMS based B-scan image is given by the time to multiply two matrices. The FT based strategy is obviously faster in terms of producing a B-scan, when no data preparation is required before FFT. Indeed, in principle, for $N_{k}$ points, a FFT based B-scan requires $N_{x} N_{k} \log _{2} N_{k}$ operations, while a MS based B-scan obtained by matrix multiplication $N_{x} N_{z}\left(2 N_{k}-1\right)$ operations. To obtain the same axial length image in both methods, $N_{z}=N_{k} / 2$, in which case the FFT method is a clear winner. However, in practice the channeled spectra have to be prepared before FFT, i.e. data need to be organized in equal wavenumber slots that requires extra time and resources. In addition, images in the CMS-OCT, 
that use $N_{z}$ less than $N_{k} / 2$ can be quicker produced than using the FFT based OCT. For such cases, the time to produce Bscans via Eq. (7) is similar or shorter than the time required by FFT-OCT with resampling.

\section{BENCHMARKING}

To evaluate the conditions in which the CMS method can provide real-time cross-sectional images, we compared the time to produce a B-scan image using the CMS method with the time required to produce the same image via FFTs, when data was resampled only but not compensated for unbalanced dispersion. Our findings are summarized in Fig. 2.

To perform the benchmarking, a LabVIEW 2015 project was created to run on a computer equipped with an Intel I75960X @ 3.0 GHz octacore processor (2 logical cores per physical core) and 16 GB of RAM. Each B-scan is built using $\mathrm{Nx}=1000$ A-scans. Two situations are considered for the CMS case, $\mathrm{N}_{\mathrm{z}}=\mathrm{N}_{\mathrm{k}} / 2$ to mimic the axial size of the B-scan obtained in FFT based OCT and also a smaller number of sampled points, $\mathrm{N}_{\mathrm{z}}=\mathrm{N}_{\mathrm{k}} / 4$. For the conventional case of resampled FFT method (FFTR) (solid circles), data were resampled via a cubic B-spline interpolation procedure before FFT. The FFT requires $\mathrm{N}_{\mathrm{x}} \mathrm{N}_{\mathrm{k}} \log _{2} \mathrm{~N}_{\mathrm{k}}$ operations. When using the CMS method, for the same number of $\mathrm{N}_{\mathrm{z}}=\mathrm{N}_{\mathrm{k}} / 2$ axial points, a number of $\mathrm{N}_{\mathrm{x}} \mathrm{N}_{\mathrm{k}}\left(2 \mathrm{~N}_{\mathrm{k}}-1\right)$ operations are required. Therefore, the time to produce a B-scan using the FFT approach is shorter than the time to produce a CMS-OCT B-scan image. This curve was obtained using the standard procedure of multiplying matrices. However, by using the Multicore Analysis \& Sparse Matrix toolkit (MASMT), the time performance of multiplying matrices is tremendously improved.

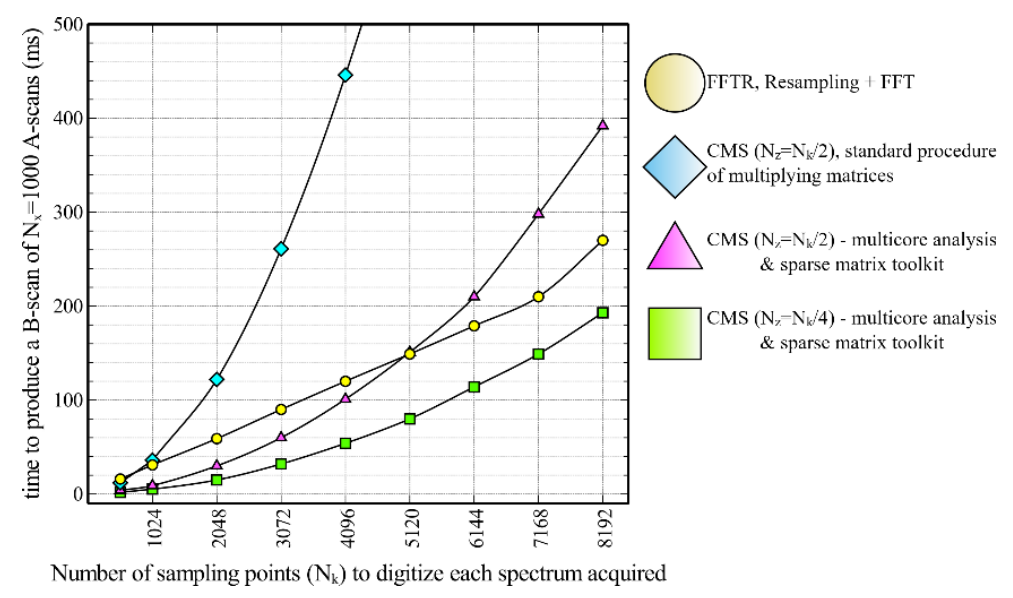

Figure 2. Time to produce a cross-sectional image (B-scan) using both methods, FFT and MS vs the number of sampling points used to digitize each channeled spectrum. Each B-scan is built from $\mathrm{N}_{\mathrm{x}}=1000$ A-scans

As showed in Fig. 2, for small values of $\mathrm{N}_{\mathrm{k}}$, (up to around 5120), the CMS technique with the adequate LabVIEW toolkit can produce B-scans faster than the procedure using the FFT after resampling (FFTR). Obviously, if data needed to be additionally compensated for dispersion, the FFT based method would have required even longer. By running our benchmarking project on computers with different, weaker CPU specifications, but with at least 4 cores, we found out that typically, CMS is faster than FFT when $\mathrm{N}_{\mathrm{k}}<3000$. Typically, if the emphasis is not on a long axial range, there is no need for a high number of sampling points when digitizing data. For $\mathrm{N}_{\mathrm{k}}=4096$ sampling points, with $\mathrm{N}_{\mathrm{z}}=2048$ distinct points in depth, a B-scan can be produced in about $102 \mathrm{~ms}$. This does not allow B-scans to be produced during the time of the next frame when using a 50-Hz galvo-scanner. However, such a performance is faster than the FFTR method (120 ms) but insufficiently quick to categorize the operation as truly real-time.

An exquisite capability of the MS method is the possibility to reduce the time to display the image by reducing the number of depth points, $\mathrm{Nz}$. This number is not connected to $\mathrm{N}_{\mathrm{k}}$ and by reducing $\mathrm{N}_{\mathrm{z}}$, the axial resolution is not affected, the image becomes sparse only. The drawback of this operation is either a reduction of the axial range (when $\mathrm{N}_{\mathrm{z}}$ masks are produced to encode a shorter axial range than that determined by the digitizer) or a less axial resolution (when $\mathrm{N}_{\mathrm{z}}$ less dense masks are produced to cover the full axial range determined by the digitizer). This limitation is not essential in most applications. With squares, we show in Fig. 2 that for a reduced number $N_{z}=N_{k} / 4$ axial points in the A-scan (which corresponds to an axial range of around $6 \mathrm{~mm}$ if the density of masks is kept constant), the CMS technique can provide images faster than its FFTR counterpart for any number of sampling points $\mathrm{N}_{\mathrm{k}}$ used to digitize the CS. According to the benchmarking shown in Fig. 2, for the particular case when $\mathrm{N}_{\mathrm{k}}=4096$, and $\mathrm{N}_{\mathrm{z}}=\mathrm{N}_{\mathrm{k}} / 4$, a B-scan image can be produced in $52 \mathrm{~ms}$. 


\section{CONCLUSION}

By performing the MS method, it is possible to: (1) directly access information from selected depths; (ii) eliminate the process of resampling, required by the FT based conventional technology, with immediate consequences in improving the decay of sensitivity with depth, achieving the expected axial resolution limit, reduction in the time to display an image and lowering the cost of OCT assembly; (iii) OCT interferometer tolerant to dispersion left unbalanced.

It is possible to speed up the production of B-scans, as it is possible to act on the size of the axial region of interest to be imaged (ROI). In FT-OCT, this range is determined by the speed of the digitizer that also determines the number of sampling points $\mathrm{N}_{\mathrm{k}}$ to digitize each channeled spectrum. The maximum axial range is determined in both methods, conventional FFT and MS by the number of sampling points $\mathrm{N}_{\mathrm{k}}$ used to digitize each channeled spectrum. However, after acquisition, each method offers different functionality in preparing images. In FFT based OCT, the $\mathrm{N}_{\mathrm{k}}$ number of points dictates the axial extension of the B-scan OCT image. A smaller region of interest (ROI) is achievable only by cropping the final image. In MS, the axial ROI is determined by $\mathrm{N}_{\mathrm{z}}$, the number of masks to be used at the Slave stage. Consequently, it is possible to adjust the ROI easily, to match the axial size of the sample to be imaged.

The use of a large number of sampling points $\mathrm{N}_{\mathrm{k}}$ for digitization cannot be avoided in some cases, hence an increased computation time to resample data before FFT. MS-OCT solves this issue, by decoupling the axial size of the ROI from $\mathrm{N}_{\mathrm{k}}$. In FFT based OCT, as the optical axial resolution is digitally compromised when an insufficient number of points is used to digitize the channeled spectra, very often data is zero-padded before FFT. In CMS, to tackle this inconvenience, a sufficiently large number $\mathrm{N}_{\mathrm{z}}$ of axial reflectivities have to be estimated. As in CMS, the axial ROI can be decoupled from $\mathrm{N}_{\mathrm{k}}$, the number of axial reflectivities $\mathrm{N}_{\mathrm{z}}$ can be reduced up to the level where the digital resolution equals the optical resolution. In FFT based method, due to zero-padding the amount of data produced cannot be limited.

\section{ACKNOWLEDGEMENTS}

A. Bradu and A. Podoleanu acknowledge the support of ERC (http://erc.europa.eu) COGATIMABIO 249889. S. Rivet acknowledges the Marie-Curie Intra-European Fellowship for Career Development, No. 625509. A.P. is also supported by the NIHR Biomedical Research Centre at Moorfields Eye Hospital NHS Foundation Trust and the UCL Institute of Ophthalmology, by the European Industrial Doctorate UBAPHODESA, FP7-PEOPLE-2013-ITN 607627 and by the Royal Society Wolfson Research Merit Award. A Bradu is also supported by the EPSRC - EP/N019229/1.

\section{REFERENCES}

[1] Rivet, S., Maria, M., Bradu, A., T., Leick, L., and Podoleanu, A., “Complex master slave interferometry,” Opt. Express 24, 2885-2904 (2016).

[2] Podoleanu, A., and Bradu, A., "Master-slave interferometry for parallel spectral domain interferometry sensing and versatile 3D optical coherence tomography," Opt. Express 21, 19324-19338 (2013).

[3] Bradu, A., Kapinchev, K., Barnes, F., and Podoleanu, A., "On the possibility of producing true real-time retinal crosssectional images using a graphics processing unit enhanced master-slave optical coherence tomography system," J. Biomed. Opt., 20(7), 076008 (2015).

[4] Bradu, A., Kapinchev, K., Barnes, F., and Podoleanu, A., "Master slave en-face OCT/SLO,” Biomed. Opt. Express 6, 3655-3669 (2015).

[5] Bradu, A., and Podoleanu, A., "Calibration-free B-scan images produced by master/slave optical coherence tomography," Opt. Lett. 39, 450-453 (2014).

[6] Bradu, A., Maria, M., and Podoleanu, A., "Demonstration of tolerance to dispersion of Master/Slave Interferometry," Opt. Express, 23(11) 14148-14161 (2015).

[7] Bradu, A., Kapinchev, K., Barnes, F., and Podoleanu, A., "On the possibility of producing true real-time retinal crosssectional images using a graphics processing unit enhanced master-slave optical coherence tomography system," J. Biomed. Opt., 20(7), 076008 (2015).

[8] Bradu, A., Rivet, S., and Podoleanu, A., "Master/slave interferometry - ideal tool for coherence revival swept source optical coherence tomography," Biomed. Opt. Express 7, 2453-2468 (2016). 